doi: https://doi.org/10.15407/dopovidi2018.11.019

УДК 004.8

\title{
А.Ф. Кургаев
}

Институт кибернетики им. В.М. Глушкова НАН Украины, Киев

E-mail: afkurgaev@ukr.net

\section{Новое определение SPARQL - языка запросов Semantic Web}

Представлено академиком НАН Украины А.В. Палагиным

Дано в метаязыке нормальных форм знаний (НФЗ) текстовое и часть графического описания синтаксиса языка SPARQL структурированных запросов Seтапtic Web. Наличие таких описаний гарантирует реализуемость языка SPARQL с реализащией интерпретатора метаязыка НФЗ. Показано, что выразительные возможности метаязыка НФЗ для формального описания SPARQL вполне сопоставимы с выразительными возможностями метаязыка Extended Backus-Naur Form.

Ключевые слова: метаязык нормальных форм знаний, формальное описание, язык SPARQL, Semantic Web.

Для извлечения нужных данных из непрерывно возрастующих коллекций публичных и частных данных все шире используется язык запросов SPARQL, упрощающий доступ как к структурированным данным семантического веб-проекта, так и к данным на разных платформах.

SPARQL (рекурсивный акроним от англ. SPARQL Protocol and RDF Query Language) язык запросов к данным, представленным согласно модели RDF, а также протокол для передачи этих запросов и ответов на них.

SPARQL упрощает интеграцию хранилища данных на многих предприятиях за счет широкого выбора инструментов и библиотек приложений для извлечения, обновления, микширования и сопоставления RDF доступных данных [1].

Целью статьи является экспериментальное исследование выразительных возможностей метаязыка нормальных форм знаний (НФЗ) [2-5] на примере описания языка запросов SPARQL.

1. Общая характеристика SPARQL. SPARQL - это язык запросов Semantic Web, который позволяет [6-8]:

извлекать значение из структурированных и полуструктурированных данных;

исследовать данные, запрашивая неизвестные отношения;

выполнять сложные объединения разрозненных баз данных в одном простом запросе;

(C) А.Ф. Кургаев, 2018 


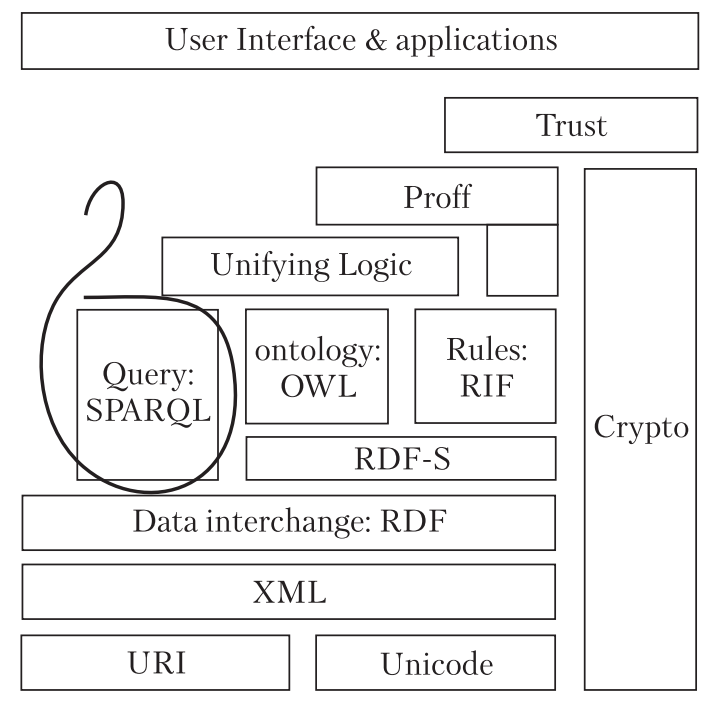

Puc. 1. Место SPARQL в семантическом стеке Тима Бернерс-Ли [9]

преобразовывать RDF данные из одного словаря в другой.

Запрос SPARQL включает в себя:

префиксные декларации для сокращения URI; определение набора данных, которого касается RDF запрос;

утверждение результата, определяющего информацию, возвращаемую из запроса;

шаблон запроса для поиска в базовом наборе данных;

модификаторы запросов, нарезку, упорядочение и другие изменения результатов запроса.

SPARQL базируется на сопоставлении графов, имеет возможности для запроса необходимых и необязательных графических паттернов (англ. pattern - образец, шаблон; форма, модель; схема, диаграмма), также поддерживает агрегацию, вложенные запросы, отрицания, создание значений с помощью выражений тестирования и исходного RDF-графа. Результатами SPARQL запросов могут быть наборы результатов или RDF графы.

SPARQL имеет четыре формы запросов:

SELECT возвращает все или подмножество переменных, связанных со значениями согласно шаблону запроса. Может вводить и новые переменные.

CONSTRUCT возвращает RDF граф, сконструированный заменой переменных в наборе триплета шаблонов.

ASK возвращает логический результат проверки наличия решения для шаблона запроса.

DESCRIBE возвращает один результирующий RDF граф, описывающий найденные ресурсы.

Ключевое слово FILTER выражает ограничение на решения по всей группе, выбранной графовым шаблоном. В частности, FILTER исключает произвольные решения, которые при подстановке в выражение приведут к эффективному булевому значению false или вызовут ошибку.

Выражения SPARQL строятся согласно грамматике и обеспечивают доступ к функциям (именованным IRI) и операторам (вызываются ключевыми словами и символами в грамматике SPARQL). Грамматика SPARQL идентифицирует набор операторов (например, $\& \&,{ }^{*}$, isIRI ), используемых для построения ограничений. Операторы SPARQL [6, разделы $17.3,17.4]$ могут использоваться для сопоставления значений типизированных литералов.

Mесто SPARQL в структуре языков Semantic Web дано на рис. 1.

2. Формальное определение языка SPARQL в метаязыке НФЗ. В данном изложении использована последняя, доступная автору, официальная редакция нормативных документов языка SPARQL, принятая консорциумом W3C [6]. В нормативном описании языка SPARQL правила с именами в верхнем регистре употребляются в качестве терминалов. B грамматику есть две точки входа: Queryunit для запросов и Updateunit для обновления. 
В приведенном ниже формальном описании языка SPARQL использованы следующие метасимволы метаязыка НФЗ $[2,3,5]$ :

«=»- разделитель, отделяет имя понятия (нетерминала) от его определения;

«; - конец определения понятия;

《» (пробел) - отношение конкатенации;

«/» - отношение альтернативного выбора;

«(«, ) - итерационные скобки обрамляют повторяемую (нуль или больше раз)

структуру понятий;

«^»- отношение отрицания примыкающего понятия;

«' - текстовая кавычка;

true - тождественно истинное понятие с пустым объемом;

знаки /* и * обрамляют комментарий.

В нотации метаязыка НФЗ текстуальное определение SPARQL дано в таблице, а первые две страницы графического определения - на рис. 2 и рис. 3.

Определение языка SPARQL в метаязыке НФЗ

\begin{tabular}{|c|c|c|c|}
\hline 1 & QueryUnit & $=$ & Query; \\
\hline 2 & Query & $=$ & Prologue Query_ValuesClause; \\
\hline $2 \mathrm{a}$ & Query_ & & SelectQuery / ConstructQuery / DescribeQuery / AskQuery; \\
\hline 3 & UpdateUnit & $=$ & Update; \\
\hline 4 & Prologue & $=$ & ( BaseDecl / PrefixDecl); \\
\hline 5 & BaseDecl & $=$ & 'BASE' IRIREF; \\
\hline 6 & PrefixDecl & $=$ & 'PREFIX' PNAME_NS IRIREF; \\
\hline 7 & SelectQuery & $=$ & SelectClause (DatasetClause) WhereClause SolutionModifier; \\
\hline 8 & SubSelect & $=$ & SelectClause WhereClause SolutionModifier ValuesClause; \\
\hline 9 & SelectClause & $=$ & 'SELECT' SelectClause_a SelectClause_b; \\
\hline $9 \mathrm{a}$ & SelectClause_a & $=$ & 'DISTINCT' / 'REDUCED' / true; \\
\hline $9 \mathrm{~b}$ & SelectClause_b & $=$ & SelectClause_c (SelectClause_c) $/{ }^{\prime *}$; \\
\hline $9 \mathrm{c}$ & SelectClause_c & $=$ & Var / '(' Expression 'AS' Var ')' ; \\
\hline 10 & ConstructQuery & $=$ & 'CONSTRUCT' ConstructQuery_a; \\
\hline $10 \mathrm{a}$ & ConstructQuery_a & $=$ & $\begin{array}{l}\text { ConstructTemplate (DatasetClause) WhereClause } \\
\text { SolutionModifier / (DatasetClause) 'WHERE' } \\
\text { '\{' ConstructQuery_b '\}' SolutionModifier ; }\end{array}$ \\
\hline $10 \mathrm{~b}$ & ConstructQuery_b & $=$ & TriplesTemplate / true; \\
\hline 11 & DescribeQuery & $=$ & $\begin{array}{l}\text { 'DESCRIBE' DescribeQuery_a (DatasetClause) } \\
\text { DescribeQuery_b SolutionModifier; }\end{array}$ \\
\hline $11 \mathrm{a}$ & DescribeQuery_a & $=$ & VarOrIri ( VarOrIri) / ‘*’; \\
\hline $11 b$ & DescribeQuery_b & $=$ & WhereClause / true; \\
\hline 12 & AskQuery & $=$ & 'ASK' (DatasetClause) WhereClause SolutionModifier; \\
\hline 13 & DatasetClause & $=$ & 'FROM' DatasetClause_a; \\
\hline $13 \mathrm{a}$ & DatasetClause_a & $=$ & DefaultGraphClause / NamedGraphClause ; \\
\hline 14 & DefaultGraphClause & $=$ & SourceSelector; \\
\hline 15 & NamedGraphClause & $=$ & 'NAMED' SourceSelector; \\
\hline 16 & SourceSelector & $=$ & iri; \\
\hline 17 & WhereClause & $=$ & WhereCl GroupGraphPattern; \\
\hline
\end{tabular}


Продолжение табл.

\begin{tabular}{|c|c|c|c|}
\hline $17 \mathrm{a}$ & WhereCl & $=$ & 'WHERE' / true; \\
\hline 18 & SolutionModifier & $=$ & SolMod_a SolMod_bSolMod_c SolMod_d; \\
\hline $18 \mathrm{a}$ & SolMod_a & $=$ & GroupClause / true; \\
\hline $18 \mathrm{~b}$ & SolMod_b & $=$ & HavingClause / true; \\
\hline $18 \mathrm{c}$ & SolMod_c & $=$ & OrderClause / true; \\
\hline $18 \mathrm{~d}$ & SolMod_d & $=$ & LimitOffsetClauses / true; \\
\hline 19 & GroupClause & $=$ & 'GROUP' 'BY' (GroupCondition); \\
\hline 20 & GroupCondition & $=$ & BuiltInCall / FunctionCall / '('Expression GrCond ')' / Var; \\
\hline $20 \mathrm{a}$ & GrCond & $=$ & 'AS' Var / true; \\
\hline 21 & HavingClause & $=$ & 'HAVING' (HavingCondition); \\
\hline 22 & HavingCondition & $=$ & Constraint; \\
\hline 23 & OrderClause & $=$ & 'ORDER' 'BY' (OrderCondition); \\
\hline 24 & OrderCondition & $=$ & OrdCond BrackettedExpression / Constraint / Var ; \\
\hline $24 \mathrm{a}$ & OrdCond & $=$ & 'ASC' / 'DESC'; \\
\hline 25 & LimitOffsetClauses & $=$ & LimitClause LimOffCl_a / OffsetClause LimOffCl_b; \\
\hline $25 \mathrm{a}$ & LimOffCl_a & $=$ & OffsetClause / true; \\
\hline $25 b$ & LimOffCl_b & $=$ & LimitClause / true; \\
\hline 26 & LimitClause & $=$ & 'LIMIT’ INTEGER; \\
\hline 27 & OffsetClause & $=$ & 'OFFSET' INTEGER; \\
\hline 28 & ValuesClause & $=$ & 'VALUES' DataBlock / true; \\
\hline 29 & Update & $=$ & Prologue Upd_a ; \\
\hline $29 \mathrm{a}$ & Upd_a & $=$ & Update1 Upd_b / true; \\
\hline $29 b$ & Upd_b & $=$ & ‘;’ Update / true; \\
\hline 30 & Update1 & $=$ & $\begin{array}{l}\text { Load / Clear / Drop / Add / Move / Copy / Create } \\
\text { / InsertData / DeleteData / DeleteWhere / Modify; }\end{array}$ \\
\hline 31 & Load & $=$ & 'LOAD' Load_a iri Load_b; \\
\hline $31 \mathrm{a}$ & Load_a & $=$ & 'SILENT' / true; \\
\hline $31 b$ & Load_b & $=$ & 'INTO' GraphRef / true; \\
\hline 32 & Clear & $=$ & 'CLEAR' Load_a GraphRefAll; \\
\hline 33 & Drop & $=$ & 'DROP’ Load_a GraphRefAll; \\
\hline 34 & Create & $=$ & 'CREATE' Load_a GraphRef; \\
\hline 35 & Add & $=$ & 'ADD’ Load_a GraphOrDefault 'TO' GraphOrDefault; \\
\hline 36 & Move & $=$ & 'MOVE' Load_a GraphOrDefault 'TO' GraphOrDefault; \\
\hline 37 & Copy & $=$ & 'COPY’ Load_a GraphOrDefault 'TO’ GraphOrDefault; \\
\hline 38 & InsertData & $=$ & 'INSERT DATA' QuadData; \\
\hline 39 & DeleteData & $=$ & 'DELETE DATA' QuadData; \\
\hline 40 & DeleteWhere & $=$ & 'DELETE WHERE' QuadPattern; \\
\hline 41 & Modify & $=$ & $\begin{array}{l}\text { Modify_a Modify_b(UsingClause) 'WHERE' } \\
\text { GroupGraphPattern; }\end{array}$ \\
\hline $41 \mathrm{a}$ & Modify_a & $=$ & 'WITH' iri / true; \\
\hline $41 b$ & Modify_b & $=$ & DeleteClause Modify_c / InsertClause ; \\
\hline $41 \mathrm{c}$ & Modify_c & $=$ & InsertClause / true; \\
\hline 42 & DeleteClause & $=$ & ‘DELETE’ QuadPattern; \\
\hline 43 & InsertClause & $=$ & 'INSERT' QuadPattern; \\
\hline 44 & UsingClause & $=$ & |'USING' UsingClause_a; \\
\hline
\end{tabular}


Продолжение табл.

\begin{tabular}{|c|c|c|c|}
\hline $44 \mathrm{a}$ & UsingClause_a & $=$ & iri / 'NAMED' iri ; \\
\hline 45 & GraphOrDefault & $=$ & 'DEFAULT' / GrOrDef iri; \\
\hline $45 \mathrm{a}$ & GrOrDef & $=$ & 'GRAPH' / true; \\
\hline 46 & GraphRef & $=$ & 'GRAPH’ iri; \\
\hline 47 & GraphRefAll & $=$ & GraphRef / 'DEFAULT' / 'NAMED' / 'ALL'; \\
\hline 48 & QuadPattern & $=$ & '\{'Quads '\}’; \\
\hline 49 & QuadData & $=$ & '\{'Quads '\}; \\
\hline 50 & Quads & $=$ & TriplTemp ( QuadsNotTriples point TriplTemp ); \\
\hline $50 \mathrm{a}$ & TriplTemp & $=$ & TriplesTemplate / true; \\
\hline $50 \mathrm{~b}$ & point & $=$ & $\because /$ true; \\
\hline 51 & QuadsNotTriples & $=$ & 'GRAPH’ VarOrIri ‘\{‘ TriplTemp '\}’; \\
\hline 52 & TriplesTemplate & $=$ & TriplesSameSubject point TriplTemp; \\
\hline 53 & GroupGraphPattern & $=$ & '\{'GroupGraphPat '\}’; \\
\hline $53 \mathrm{a}$ & GroupGraphPat & $=$ & SubSelect / GroupGraphPatternSub ; \\
\hline 54 & GroupGraphPatternSub & $=$ & TriplBlock ( GraphPatternNotTriples point TriplBlock ); \\
\hline $54 \mathrm{a}$ & TriplBlock & $=$ & TriplesBlock / true; \\
\hline 55 & TriplesBlock & $=$ & TriplesSameSubjectPath point TriplBlock; \\
\hline 56 & GraphPatternNotTriples & $=$ & $\begin{array}{l}\text { GroupOrUnionGraphPattern / OptionalGraphPattern } \\
\text { / MinusGraphPattern / GraphGraphPattern } \\
\text { / ServiceGraphPattern / Filter / Bind / InlineData; }\end{array}$ \\
\hline 57 & OptionalGraphPattern & $=$ & 'OPTIONAL' GroupGraphPattern; \\
\hline 58 & GraphGraphPattern & $=$ & 'GRAPH' VarOrIri GroupGraphPattern; \\
\hline 59 & ServiceGraphPattern & $=$ & 'SERVICE' SIL VarOrIri GroupGraphPattern; \\
\hline $59 \mathrm{a}$ & SIL & $=$ & 'SILENT' / true; \\
\hline 60 & Bind & $=$ & 'BIND' '(' Expression 'AS' Var ')'; \\
\hline 61 & InlineData & $=$ & 'VALUES' DataBlock; \\
\hline 62 & DataBlock & $=$ & InlineDataOneVar / InlineDataFull; \\
\hline 63 & InlineDataOneVar & $=$ & Var '\{'(DataBlockValue) '\}; \\
\hline 64 & InlineDataFull & $=$ & InlineDataFull_a ‘\{'( '(‘ (DataBlockValue) ')' / NIL ) '\}’; \\
\hline $64 a$ & InlineDataFull_a & $=$ & '(' (Var) ')' / NIL; \\
\hline 65 & DataBlockValue & $=$ & iri / RDFLiteral / NumericLiteral / BooleanLiteral / 'UNDEF'; \\
\hline 66 & MinusGraphPattern & $=$ & 'MINUS' GroupGraphPattern; \\
\hline 67 & GroupOrUnionGraphPattern & $=$ & GroupGraphPattern ( 'UNION’ GroupGraphPattern ); \\
\hline 68 & Filter & $=$ & 'FILTER' Constraint; \\
\hline 69 & Constraint & $=$ & BrackettedExpression / BuiltInCall / FunctionCall; \\
\hline 70 & FunctionCall & $=$ & iri ArgList; \\
\hline 71 & ArgList & $=$ & '(‘ DIST Expression (','Expression ) ')' / NIL; \\
\hline $71 \mathrm{a}$ & DIST & $=$ & 'DISTINCT' / true ; \\
\hline 72 & ExpressionList & $=$ & '(' Expression ( ',' Expression ) ')' / NIL; \\
\hline 73 & ConstructTemplate & $=$ & '\{“ ConstrTempl '\}’; \\
\hline $73 \mathrm{a}$ & ConstrTempl & $=$ & ConstructTriples / true; \\
\hline 74 & ConstructTriples & $=$ & TriplesSameSubject point ConstrTempl; \\
\hline 75 & TriplesSameSubject & $=$ & VarOrTerm PropertyListNotEmpty / TriplesNode PropertyList; \\
\hline 76 & PropertyList & $=$ & PropList; \\
\hline $76 \mathrm{a}$ & PropList & $=$ & PropertyListNotEmpty / true; \\
\hline
\end{tabular}


Продолжение табл.

\begin{tabular}{|c|c|c|c|}
\hline 77 & PropertyListNotEmpty & $=$ & Verb ObjectList ( ‘; VerbObjList ); \\
\hline $77 \mathrm{a}$ & VerbObjList & $=$ & Verb ObjectList / true; \\
\hline 78 & Verb & $=$ & VarOrIri / 'a'; \\
\hline 79 & ObjectList & $=$ & Object ( ',' Object ); \\
\hline 80 & Object & $=$ & GraphNode; \\
\hline 81 & TriplesSameSubjectPath & $=$ & $\begin{array}{l}\text { VarOrTerm PropertyListPathNotEmpty } \\
\text { / TriplesNodePath PropertyListPath; }\end{array}$ \\
\hline 82 & PropertyListPath & $=$ & PropertyListPathNotEmpty / true; \\
\hline 83 & PropertyListPathNotEmpty & $=$ & VerbPathSimple_a ObjectListPath ( ‘’ VerbPathSimple_b ); \\
\hline $83 \mathrm{a}$ & VerbPathSimple_a & $=$ & VerbPath / VerbSimple ; \\
\hline $83 b$ & VerbPathSimple_b & $=$ & VerbPathSimple_a ObjectList / true; \\
\hline 84 & VerbPath & $=$ & Path; \\
\hline 85 & VerbSimple & $=$ & Var; \\
\hline 86 & ObjectListPath & $=$ & ObjectPath ( ', ObjectPath ); \\
\hline 87 & ObjectPath & $=$ & GraphNodePath; \\
\hline 88 & Path & $=$ & PathAlternative; \\
\hline 89 & PathAlternative & $=$ & PathSequence ('/' PathSequence ); \\
\hline 90 & PathSequence & $=$ & PathEltOrInverse ( '/' PathEltOrInverse ); \\
\hline 91 & PathElt & $=$ & PathPrimary PathMod_a; \\
\hline $91 \mathrm{a}$ & PathMod_a & $=$ & PathMod / true; \\
\hline 92 & PathEltOrInverse & $=$ & PathElt / '^' PathElt; \\
\hline 93 & PathMod & $=$ & '?' / /*' / '+'; \\
\hline 94 & PathPrimary & $=$ & iri / 'a' / '!' PathNegatedPropertySet / '(' Path ')'; \\
\hline 95 & PathNegatedPropertySet & $=$ & PathOneInPropertySet / '(' PathNegatedPropertySet_a ')'; \\
\hline $95 \mathrm{a}$ & PathNegatedPropertySet_a & $=$ & PathOneInPropertySet ( '/' PathOneInPropertySet ) / true; \\
\hline 96 & PathOneInPropertySet & $=$ & PathOneInPropertySet_a / ‘`’ PathOneInPropertySet_a; \\
\hline $96 \mathrm{a}$ & PathOneInPropertySet_a & $=$ & iri / 'a'; \\
\hline 97 & Integer & $=$ & INTEGER; \\
\hline 98 & TriplesNode & $=$ & Collection / BlankNodePropertyList; \\
\hline 99 & BlankNodePropertyList & $=$ & '[' PropertyListNotEmpty ']’; \\
\hline 100 & TriplesNodePath & $=$ & CollectionPath / BlankNodePropertyListPath; \\
\hline 101 & BlankNodePropertyListPath & $=$ & '[' PropertyListPathNotEmpty ']'; \\
\hline 102 & Collection & $=$ & '(' GraphNode (GraphNode) ')'; \\
\hline 103 & CollectionPath & $=$ & '(' GraphNodePath (GraphNodePath) ')'; \\
\hline 104 & GraphNode & $=$ & VarOrTerm / TriplesNode; \\
\hline 105 & GraphNodePath & $=$ & VarOrTerm / TriplesNodePath; \\
\hline 106 & VarOrTerm & $=$ & Var / GraphTerm; \\
\hline 107 & VarOrIri & $=$ & Var / iri; \\
\hline 108 & Var & $=$ & VAR1 / VAR2; \\
\hline 109 & GraphTerm & $=$ & $\begin{array}{l}\text { iri / RDFLiteral / NumericLiteral / BooleanLiteral } \\
\text { / BlankNode / NIL; }\end{array}$ \\
\hline 110 & Expression & $=$ & ConditionalOrExpression; \\
\hline 111 & ConditionalOrExpression & $=$ & $\begin{array}{l}\text { ConditionalAndExpression } \\
\text { ('"l' ConditionalAndExpression ); }\end{array}$ \\
\hline 112 & ConditionalAndExpression & $=$ & ValueLogical ( '\&\&' ValueLogical ); \\
\hline
\end{tabular}


Продолжение табл.

\begin{tabular}{|c|c|c|c|}
\hline 113 & ValueLogical & $=$ & RelationalExpression; \\
\hline 114 & RelationalExpression & $=$ & NumericExpression RelationalExpression_a; \\
\hline $114 \mathrm{a}$ & RelationalExpression_a & $=$ & $\begin{array}{l}\text { '=' NumericExpression / '!=' NumericExpression } \\
\text { / '<' NumericExpression / '>' NumericExpression } \\
\text { /'<=' NumericExpression / >=' NumericExpression } \\
\text { / 'IN' ExpressionList / 'NOT' 'IN' ExpressionList / true; }\end{array}$ \\
\hline 115 & NumericExpression & $=$ & AdditiveExpression; \\
\hline 116 & AdditiveExpression & $=$ & $\begin{array}{l}\text { MultiplicativeExpression ( '+' MultiplicativeExpression } \\
\text { /'-' MultiplicativeExpression / NumLiteral } \\
\text { ( '*' UnaryExpression / ‘/' UnaryExpression ) ); }\end{array}$ \\
\hline $116 \mathrm{a}$ & NumLiteral & $=$ & NumericLiteralPositive / NumericLiteralNegative; \\
\hline 117 & MultiplicativeExpression & $=$ & $\begin{array}{l}\text { UnaryExpression ( '*’ UnaryExpression } \\
/ / / \text { UnaryExpression ); }\end{array}$ \\
\hline 118 & UnaryExpression & $=$ & $\begin{array}{l}\text { '?’ PrimaryExpression / ‘+’ PrimaryExpression } \\
\text { /'-' PrimaryExpression / PrimaryExpression; }\end{array}$ \\
\hline 119 & PrimaryExpression & $=$ & $\begin{array}{l}\text { BrackettedExpression / BuiltInCall / iriOrFunction } \\
\text { / RDFLiteral / NumericLiteral / BooleanLiteral / Var; }\end{array}$ \\
\hline 120 & BrackettedExpression & $=$ & '('Expression ')'; \\
\hline 121 & BuiltInCall & $=$ & BuiltInCall_1 / BuiltInCall_2; \\
\hline $121 \mathrm{a}$ & BuiltInCall_1 & $=$ & 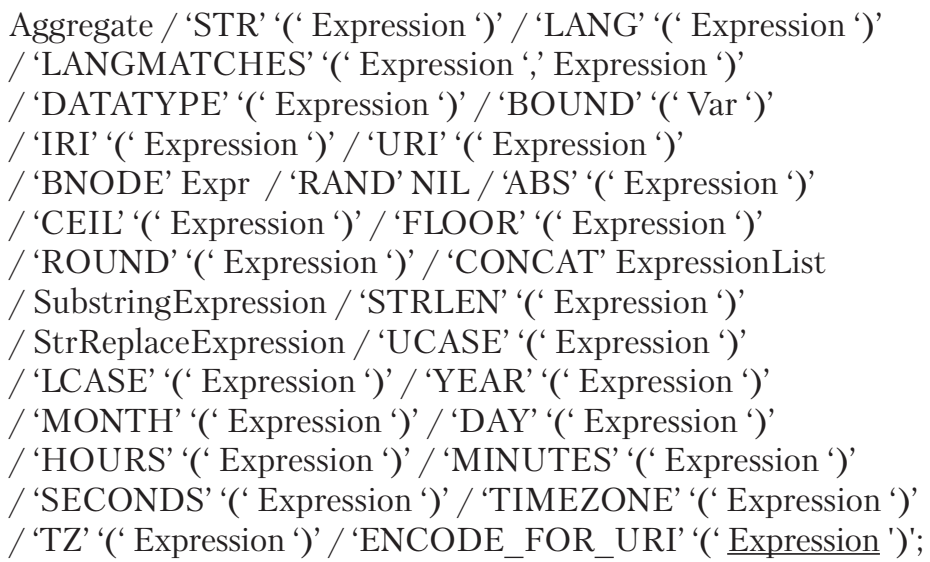 \\
\hline $121 b$ & BuiltInCall_2 & & $\begin{array}{l}\text { 'NOW' NIL / 'UUID' NIL / 'STRUUID' NIL } \\
\text { / 'MD5' '(' Expression ')' / 'SHA1' '(' Expression ')' } \\
\text { / 'SHA256' '(' Expression ')' / 'SHA384' '(' Expression ' ') } \\
\text { / 'SHA512' '(' Expression ')' / ' 'COALESCE' ExpressionList } \\
\text { / 'isIRI' '(' Expression ')' / 'isURI' '(' Expression ')' } \\
\text { / 'isBLANK' '(' Expression ')' / 'isNUMERIC' '(' Expression ')' } \\
\text { / 'CONTAINS' '(' Expression ',' Expression ' ')' } \\
\text { / 'STRSTARTS' '(' Expression ',' Expression ')' } \\
\text { / 'STRENDS' '(' Expression ',' Expression ' ')' } \\
\text { / 'STRBEFORE' '(' Expression ',' Expression ' ')' } \\
\text { / 'STRAFTER' '(' Expression ',' Expression ')' } \\
\text { / 'IF' '(' Expression ',' Expression ',' Expression ')' } \\
\text { / 'STRLANG' '(' Expression ',' Expression ')' } \\
\text { / 'STRDT' '(' Expression ',' Expression ')' } \\
\text { / 'sameTerm' '(' Expression ',' Expression ')' / RegexExpression / } \\
\text { ExistsFunc / NotExistsFunc; }\end{array}$ \\
\hline
\end{tabular}


Продолжение табл.

\begin{tabular}{|c|c|c|c|}
\hline $121 \mathrm{c}$ & Expr & $=$ & '('Expression ')' / NIL; \\
\hline 122 & RegexExpression & $=$ & 'REGEX’ '('Expression ', Expression Express ')'; \\
\hline $122 \mathrm{a}$ & Express & $=$ & ', Expression / true; \\
\hline 123 & SubstringExpression & $=$ & 'SUBSTR' '(' Expression ', Expression Express ')'; \\
\hline 124 & StrReplaceExpression & $=$ & $\begin{array}{l}\text { ‘REPLACE’ '(' Expression ‘,' Expression ', Expression } \\
\text { Express ')'; }\end{array}$ \\
\hline 125 & ExistsFunc & $=$ & 'EXISTS' GroupGraphPattern; \\
\hline 126 & NotExistsFunc & $=$ & 'NOT’ ‘EXISTS’ GroupGraphPattern; \\
\hline 127 & Aggregate & $=$ & 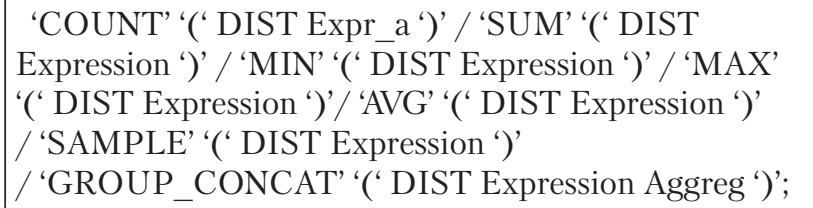 \\
\hline $127 \mathrm{a}$ & Aggreg & $=$ & ';' 'SEPARATOR' ‘=’ String / true; \\
\hline $127 \mathrm{~b}$ & Expr_a & $=$ & ‘*’ / Expression ; \\
\hline 128 & iriOrFunction & $=$ & iri ArgList / iri; \\
\hline 129 & RDFLiteral & $=$ & String RDFLit; \\
\hline $129 \mathrm{a}$ & RDFLit & $=$ & LANGTAG / ‘^^’ iri / true; \\
\hline 130 & NumericLiteral & $=$ & $\begin{array}{l}\text { NumericLiteralUnsigned / NumericLiteralPositive } \\
\text { / NumericLiteralNegative; }\end{array}$ \\
\hline 131 & NumericLiteralUnsigned & $=$ & INTEGER / DECIMAL / DOUBLE; \\
\hline 132 & NumericLiteralPositive & $=$ & $\begin{array}{l}\text { INTEGER_POSITIVE / DECIMAL_POSITIVE } \\
\text { / DOUBLE_POSITIVE; }\end{array}$ \\
\hline 133 & NumericLiteralNegative & $=$ & $\begin{array}{l}\text { INTEGER_NEGATIVE / DECIMAL_NEGATIVE } \\
\text { / DOUBLE_NEGATIVE; }\end{array}$ \\
\hline 134 & BooleanLiteral & $=$ & 'true' / 'false'; \\
\hline 135 & String & $=$ & $\begin{array}{l}\text { STRING_LITERAL1 / STRING_LITERAL2 } \\
\text { / STRING_LITERAL_LONG1 } \\
\text { / STRING_LITERAL_LONG2; }\end{array}$ \\
\hline 136 & iri & $=$ & IRIREF / PrefixedName; \\
\hline 137 & PrefixedName & $=$ & PNAME_LN / PNAME_NS; \\
\hline 138 & $\begin{array}{l}\text { BlankNode } \\
\text { Продукции для терминалов }\end{array}$ & $=$ & BLANK_NODE_LABEL / ANON; \\
\hline 139 & IRIREF & $=$ & '<'(^IRIREF_a IRIREF_b) ‘>'; \\
\hline $139 \mathrm{a}$ & IRIREF_a & $=$ & {$[\# x 00-\# x 20]$} \\
\hline $139 b$ & IRIREF_b & $=$ & {$\left[{ }^{\wedge}<>"\{\}\right] /\left[\left[^{\wedge} \backslash\right] ;\right.$} \\
\hline 140 & PNAME_NS & $=$ & PN_PREFIX ‘' / ‘’’; \\
\hline 141 & PNAME_LN & $=$ & PNAME_NS PN_LOCAL; \\
\hline 142 & BLANK_NODE_LABEL & $=$ & ‘ ' BL_NODE_LAB (PN_CHARS /'?) ; \\
\hline $142 \mathrm{a}$ & BL_NODE_LAB & $=$ & PN_CHARS_U / [0-9]; \\
\hline 143 & VAR1 & $=$ & '?' VARNAME; \\
\hline 144 & VAR2 & $=$ & '\$’ VARNAME; \\
\hline 145 & LANGTAG & $=$ & '@’ [a-zA-Z] ([a-zA-Z]) (‘-’ [a-zA-Z0-9] ([a-zA-Z0-9])); \\
\hline 146 & INTEGER & $=$ & {$[0-9]([0-9])$} \\
\hline 147 & DECIMAL & $=$ & ([0-9]) '.' [0-9] ([0-9]); \\
\hline
\end{tabular}


Окончание табл.

\begin{tabular}{|c|c|c|c|}
\hline 148 & DOUBLE & $=$ & $\begin{array}{l}{[0-9]([0-9]) ` ’([0-9]) \text { EXPONENT / ‘’ }[0-9]([0-9])} \\
\text { EXPONENT / [0-9] ([0-9]) EXPONENT; }\end{array}$ \\
\hline 149 & INTEGER_POSITIVE & $=$ & '+’ INTEGER; \\
\hline 150 & DECIMAL_POSITIVE & $=$ & '+’' DECIMAL; \\
\hline 151 & DOUBLE_POSITIVE & $=$ & ‘+’ DOUBLE; \\
\hline 152 & INTEGER_NEGATIVE & $=$ & '-' INTEGER; \\
\hline 153 & DECIMAL_NEGATIVE & $=$ & '- DECIMAL; \\
\hline 154 & DOUBLE_NEGATIVE & $=$ & '-’ DOUBLE; \\
\hline 155 & EXPONENT & $=$ & {$[\mathrm{eE}] \operatorname{sign}[0-9]([0-9]) ;$} \\
\hline $155 \mathrm{a}$ & sign & $=$ & {$[+-] /$ true; } \\
\hline 156 & STRING_LITERAL1 & $=$ & “"” ([^\#x27\#x5C\#xA\#xD] / ECHAR ) “"”; \\
\hline 157 & STRING_LITERAL2 & $=$ & '”' ([^\#x22\#x5C\#xA\#xD]/ECHAR) '”; \\
\hline 158 & STRING_LITERAL_LONG1 & $=$ & “"”’” (STR_LIT_LONG1_a STR_LIT_LONG1_b) “"””; \\
\hline $158 \mathrm{a}$ & STR_LIT_LONG1_a & $=$ & “"” / “"” / true; \\
\hline $158 \mathrm{~b}$ & STR_LIT_LONG1_b & $=$ & {$\left[{ }^{\wedge} \backslash\right] /$ ECHAR ; } \\
\hline 159 & STRING_LITERAL_LONG2 & $=$ & “"”’”' (STR_LIT_LONG2_a STR_LIT_LONG2_b) ‘"’”’; \\
\hline $159 \mathrm{a}$ & STR_LIT_LONG2_a & $=$ & '”' / '”"'”/ true; \\
\hline $159 b$ & STR_LIT_LONG2_b & $=$ & {$\left[{ }^{\wedge} \gg\right] /$ ECHAR ; } \\
\hline 160 & ECHAR & $=$ & $\vee ’[$ tbnrf $\ "]$ \\
\hline 161 & NIL & $=$ & '('(WS) ')'; \\
\hline 162 & WS & $=$ & $\# \times 20 / \# x 9 / \# x D / \# x A ;$ \\
\hline 163 & ANON & $=$ & '['(WS) ']'; \\
\hline 164 & PN_CHARS_BASE & $=$ & $\begin{array}{l}{[\mathrm{A}-\mathrm{Z}] /[\mathrm{a}-\mathrm{z}] /[\# \mathrm{x} 00 \mathrm{C} 0-\# \mathrm{x} 00 \mathrm{D} 6] /[\# \mathrm{x} 00 \mathrm{D} 8-\# \mathrm{x} 00 \mathrm{~F} 6]} \\
/[\# \mathrm{x} 00 \mathrm{~F} 8-\# \mathrm{x} 02 \mathrm{FF}] /[\# \mathrm{x} 0370-\# \mathrm{x} 037 \mathrm{D}] \\
/[\# \mathrm{x} 037 \mathrm{~F}-\# \mathrm{x} 1 \mathrm{FFF}] /[\# \mathrm{x} 200 \mathrm{C}-\# \mathrm{x} 200 \mathrm{D}] \\
/[\# \mathrm{x} 2070-\# \mathrm{x} 218 \mathrm{~F}] /[\# \mathrm{x} 2 \mathrm{C} 00-\# \mathrm{x} 2 \mathrm{FEF}] \\
/[\# \mathrm{x} 3001-\# \mathrm{xD} 7 \mathrm{FF}] /[\# \mathrm{xF} 900-\# \mathrm{xFDCF}] \\
/[\# \mathrm{xFDF} 0-\# \mathrm{xFFFD}] /[\# \mathrm{x} 10000-\# \mathrm{xEFFF}]\end{array}$ \\
\hline 165 & PN_CHARS_U & $=$ & PN_CHARS_BASE /'_'; \\
\hline 166 & VARNAME & $=$ & $\begin{array}{l}\text { BL_NODE_LAB ( PN_CHARS_U / [0-9] } \\
/ \# \text { - } 000 B 7 /[\# \mathrm{x} 0300-\# \mathrm{x} 036 \mathrm{~F}] /[\# \mathrm{x} 203 \mathrm{~F}-\# \mathrm{x} 2040])\end{array}$ \\
\hline 167 & PN_CHARS & $=$ & $\begin{array}{l}\text { PN_CHARS_U / '-' / [0-9] / \#x00B7 } \\
/[\# \times \text { x0300-\#x036F] / [\#x203F-\#x2040]; }\end{array}$ \\
\hline 168 & PN_PREFIX & $=$ & PN_CHARS_BASE PN_PR; \\
\hline $168 \mathrm{a}$ & PN_PR & $=$ & (PN_CHARS / '’) PN_CHARS / true; \\
\hline 169 & PN_LOCAL & $=$ & PN_LOC_a (PN_CHARS / '? / '? / PLX) PN_LOC_b; \\
\hline $169 \mathrm{a}$ & PN_LOC_a & $=$ & PN_CHARS_U / ' :’ [0-9] / PLX ; \\
\hline $169 \mathrm{~b}$ & PN_LOC_b & $=$ & PN_CHARS / ‘'’ PLX / true; \\
\hline 170 & PLX & $=$ & PERCENT / PN_LOCAL_ESC; \\
\hline 171 & PERCENT & $=$ & '\%' HEX HEX; \\
\hline 172 & HEX & $=$ & {$[0-9] /[A-F] /[a-f] ;$} \\
\hline 173 & PN_LOCAL_ESC & $=$ & 'YPN_LOC_ESC; \\
\hline $173 a$ & PN_LOC_ESC & $=$ & 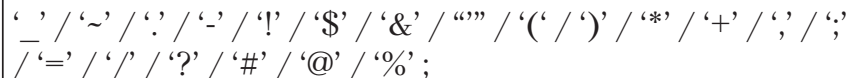 \\
\hline
\end{tabular}




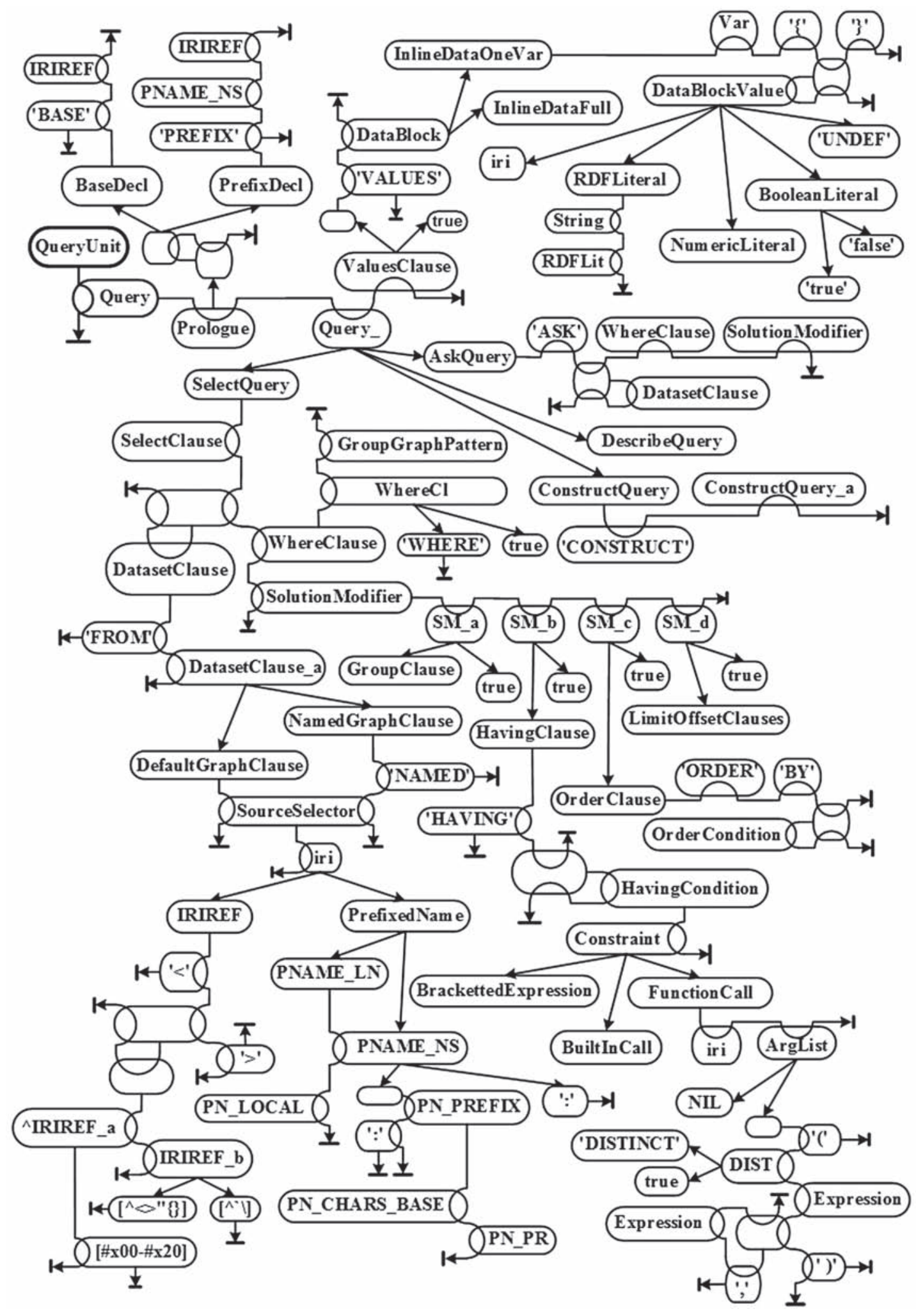

Puc. 2. Первая страница графического описания в метаязыке HФЗ синтаксиса языка SPARQL 


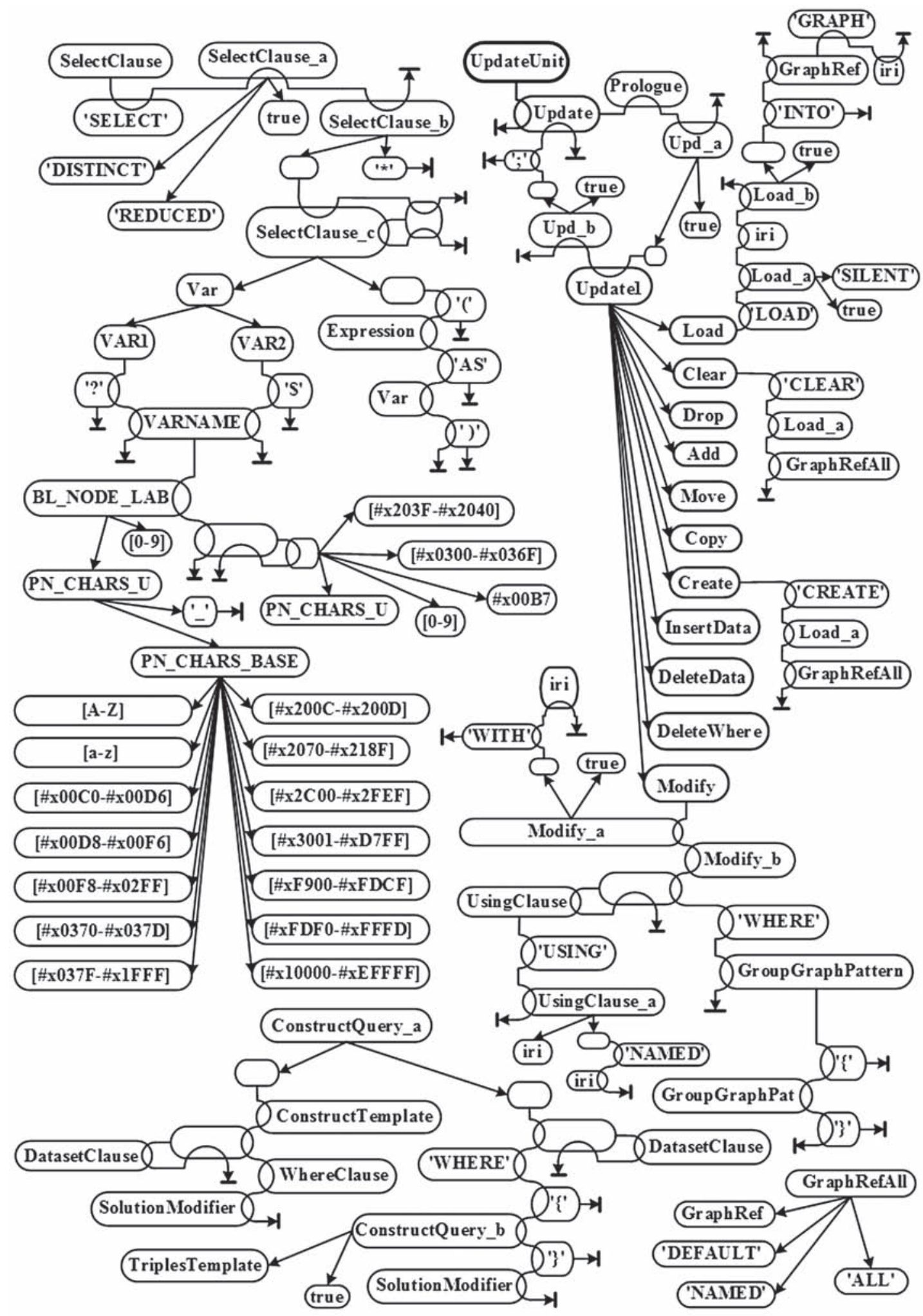

Puc. 3. Вторая страница графического описания в метаязыке НФЗ синтаксиса языка SPARQL 
3. Сопоставление предлагаемого с нормативным описанием SPARQL. Нормативное описание синтаксиса языка SPARQL метаязыком EBNF включает 173 [6], а аналогичное описание метаязыком НФЗ (см. таблицу) - 236 продукций. Дополнительные правила НФЗ-описания языка SPARQL использованы для моделирования:

структурных скобок 20 правил: 2a, 9c, 10a, 13a, 24a, 41b, 44a, 53a, 64a, 83a, 96a, 114a, 116a, 121c, 127b, 142a, 158b, 159b, 169a, 173a;

необязательности 36 правил: 9a, 10b, 17a, 18a, 18b, 18c, 18d, 20a, 25a, 25b, 29a, 29b, 31a, 31b, 41a, 41c, 45a, 50a, 50b, 54a, 59a, 71a, 73a, 76a, 77a, 83b, 91a, 95a, 122a, 127a, 129a, 155a, 158a, 159a, 168a, 169b;

одного или большего числа вхождений нетерминала и структурных скобок - правило 9b; отрицания понятий два правила 139a, 139b;

для упрощения изображения графа два правила 121a, $121 \mathrm{~b}$.

Главным результатом сопоставления рассмотренных описаний является

Утверждение 1. Выразительных возможностей метаязыка НФЗ достаточно для формального описания языка SPARQL запросов Semantic Web.

Таким образом исследованы выразительные возможности метаязыка НФЗ по отношению к нормативному описанию синтаксиса языка запросов SPARQL. Даны формальные текстовое и первые две страницы графического описания этого языка, чье наличие гарантирует реализуемость языка SPARQL с реализацией интерпретатора метаязыка НФЗ. Показано, что выразительные возможности метаязыка НФЗ для формального описания языка SPARQL близки выразительным возможностям метаязыка Extended Backus-Naur Form, уступая ему по числу правил из-за отсутствия структурных скобок и метасимвола необязательности.

\section{ЦИТИРОВАННАЯ ЛИТЕРАТУРА}

1. DuCharme B. Learning SPARQL, 2nd Edition. USA: O’Reilly Media, Inc. 2013. 386 p.

2. Кургаев А.Ф., Григорьев С.Н. Нормальные формы знаний. Допов. Нац. акад. наук Укр. 2015. № 11. C. $36-43$.

3. Кургаев А.Ф., Григорьев С.Н. Метаязык нормальных форм знаний. Кибернетика и системный анализ. 2016. 52, № 6. C. 11-20. doi: http://link.springer.com/article/10.1007/s10559-016-9885-3

4. Кургаев А.Ф., Григорьев С.Н. Интерпретатор универсальной машины Тьюринга. Допов. Нац. акад. наук Укр. 2016. № 10. С. 30-36. doi: http://dx.doi.org/10.15407/dopovidi2016.10.030

5. Кургаев А.Ф., Григорьев С.Н. Определение формальных языков в метаязыке нормальных форм знаний. Проблеми програмування. 2017. № 4. С. 37-50.

6. SPARQL 1.1: Query Language. W3C Recommendation 21 March 2013. URL: http://www.w3.org/TR/2013/ REC-sparql11-query-20130321/. (Дата обращения: 01.02.2018)

7. Feigenbaum L. SPARQL By Example: A Tutorial. VP Technology \& Standards, Cambridge Semantics. URL: http://www.cambridgesemantics.com/2008/09/sparql-by-example/. (Дата звернення: 01.02.2018)

8. Introduction aux RDF \& SPARQL: Training Module 1.3. Open Data Support: 2014 European Commission. URL: https://www.europeandataportal.eu/sites/default/files/d2.1.2_training_module_1.3_introduction_ to_rdf_sparql_en_edp.pdf. (Дата обращения: 01.02.2018)

9. Berners-Lee T., Hall W., Hendler J.A., O'Hara K., Shadbolt N., Weitzner D.J. A Framework for Web Science. Foundations and Trends in Web Science. 2006. 1, № 1. P. 1-130. doi: https://doi.org/10.1561/1800000001

Поступило в редакцию 15.05.2018 


\section{REFERENCES}

1. DuCharme, B. (2013). Learning SPARQL, 2nd Edition. USA: O’Reilly Media, Inc.

2. Kurgaev, A., Grygoryev, S. (November 2015). The normal forms of knowledge. Dopov. Nac. akad. nauk. Ukr., No. 11, pp. 36-43 (in Russian).

3. Kurgaev, A., Grygoryev, S. (November 2016). Metalanguage of Normal Forms of Knowledge. Cybernetics and Systems Analysis. 52, No. 6, pp. 839-848. doi: http://link.springer.com/article/10.1007/s10559-016-9885-3

4. Kurgaev, A. \& Grygoryev, S. (October 2016). The universal turing machine interpreter. Dopov. Nac. akad. nauk. Ukr., No. 10, pp. 30-36 (in Russian). doi: http://dx.doi.org/10.15407/dopovidi2016.10.030

5. Kurgaev, A. \& Grygoryev, S. (2017). The definition of formal languages in the meta language of normal forms of knowledge. Programming problems. No. 4, pp. 37-50 (in Russian).

6. SPARQL 1.1: Query Language. W3C Recommendation 21 March 2013. Retrieved from: http://www.w3.org/ TR/2013/REC-sparql11-query-20130321/

7. Lee Feigenbaum. SPARQL By Example: A Tutorial. VP Technology \& Standards, Cambridge Semantics. Retrieved from: http://www.cambridgesemantics.com/2008/09/sparql-by-example/.

8. Introduction aux RDF \& SPARQL: Training Module 1.3. Open Data Support: 2014 European Commission. R etrieved from: https://www.europeandataportal.eu/sites/default/files/d2.1.2_training_module_1.3_introduction_to_rdf_sparql_en_edp.pdf.

9. Berners-Lee, T., Hall, W., Hendler, J. A., O’Hara, K., Shadbolt, N. \& Weitzner, D. J. (2006) A Framework for Web Science. Foundations and Trends in Web Science, 1, No. 1, pp. 1-130. doi: https://doi.org/10.1561/ 1800000001

Received 15.05.2018

\section{О.П. Кургаєв}

Інститут кібернетики ім. В.М. Глушкова НАН України, Київ

E-mail: afkurgaev@ukr.net

НОВЕ ВИЗНАЧЕННЯ SPARQL -

МОВИ ЗАПИТІВ SEMANTIC WEB

Дано у метамові нормальних форм знань (НФЗ) текстовий і частина графічного опису синтаксису мови SPARQL структурованих запитів Semantic Web. Наявність таких описів гарантує реалізуємість мови SPARQL з реалізацією інтерпретатора метамови НФЗ. Показано, що виразні можливості метамови НФЗ для формального опису SPARQL цілком порівнянні з виразними можливостями метамови Extended Backus-Naur Form.

Ключові слова: метамова нормальних форм знань, формальний опис, мова SPARQL, Semantic Web.

\section{A.F. Kurgaev}

V.M. Glushkov Institute of Cybernetics of the NAS of Ukraine, Kiev

E-mail: afkurgaev@ukr.net

\section{NEW DEFINITION OF THE SPARQL - QUERY LANGUAGE FOR THE SEMANTIC WEB}

The text and the part of a graphic description of the syntax of the SPARQL structured Query Language for the Semantic Web are described in the metalanguage of normal forms of knowledge. These descriptions guarantee that the SPARQL language can be implemented, when the interpreter of the metalanguage of normal forms of knowledge is implemented. It is demonstrated that the expressive possibilities of the metalanguage of normal forms of knowledge for the formal description of the SPARQL language are quite comparable with those of the Extended Backus-Naur Form Metalanguage.

Keywords: metalanguage of normal forms of knowledge, formal description, SPARQL language, Semantic Web. 